the plane among the loosely looped molecules. The effect of this constraint is permanently to separate the molecules into several different categories-there are those which lie entirely on one side of the rod, those which lie entirely on the other, and those which are looped about the rod once, twice and several times. But each molecule is a kind of random walk with fixed end points and a definite number of paces. For the simple two-dimensional problem, Edwards shows how it is possible to calculate the probability that a random walk will generate analogues of the molecules in the several distinct topological classes, which is something of a triumph in itself. Each probability, of course, is a function of the end points of the molecules and of the point at which the rod-like constraint is made to intervene. It is no surprise that the entropy of the system as a whole turns out to differ from that of an unconstrained system by the addition of terms of the form $N k P \log P$, where $P$ is the probability that a random walk will yield molecules in a certain topological class and where there is one term for each class.

The great interest of the calculation is that it provides a general expression for the entropy of a constrained system in which the parameters of the constraint enter explicitly. On paper, at least, it is possible to calculate mechanical properties such as elastic constants. Edwards himself points out that the two-dimensional problem is unrealistic, that the threedimensional analogue is that of an assemblage of molecules threaded in various ways through a closed loop and that this is more directly analogous to real situations. The next instalment in his calculation will be worth waiting for.

\section{Neuropathology at Wye}

\section{Professor C. W. M. Adams writes :}

THE second symposium on neuropathology, organized by N. T. Blau and Helen Payling Wright, took place at Wye College from July 10 to 14 . The chief purpose of these symposia is to highlight growing points in the neurological sciences. The topics on this occasion were Schwann cells, neuroviruses, immuno-neurology, neurochemistry, developmental neurology and cerebrovascular disease.

Peripheral nerve ultrastructure was lucidly illustrated by D. N. Landon and P. K. Thomas. We learnt that Schwann cell cytoplasm extends into the SchmittLanterman cleft. Discussion also centred on the role of the Schwannian paranodal apparatus and on possible cation-binding by acid mucopolysaccharide in the internodal gap. This last theme was continued by C. J. Duncan, who suggested that the initial component in nerve conduction is mediated by $\mathrm{Ca}^{++}$. activated ATPase, whereas the axon potential is propagated by an ion-sensitive protein. J. B. Cavanagh discussed Schwann cell injury: diphtheria toxin inhibits certain of the cell's metabolic activities, whereas $\mathrm{X}$-irradiation impairs its proliferative activity.

The concept of "slow virus" in neurological disease was reviewed by A. P. Waterson, while J. A. Dudgeon showed that viruses persist in tissues for long periods after initial infection. Transmission of virus along Schwann cells appeared, from F. Kingsley Saunders' experimental work, to be a likely route by which an encephalitis virus enters the brain. Within the brain H. E. Webb concluded that Langat virus directly attacks neurones, while hypersensitivity is responsible for perivascular inflammation and oedema. The scrapie agent remains as enigmatic as ever; I. $H$. Pattison wondered whether it might be a relatively simple peptide quite unrelated to virus.

In experimental allergic encephalomyelitis S. Leibowitz reported that grossly increased vascular permeability is associated with perivascular inflammation, even though slight leakage occurs in the absence of local cellular infiltration. He showed that simultaneous injection of anti-lymphocytic serum slows down the immunological reaction, so that true demyelinating lesions appear even in the guinea-pig. D. Hughes illustrated the demyelinating and gliotoxic effect of multiple sclerosis serum. Nevertheless, similar results were obtained in motor neurone disease and in a quarter of control subjects. Continuing the immunological theme, the presence of antibrain antibody was demonstrated by $\mathrm{P}$. C. Wilkinson in the serum of patients with carcinomatous sensory neuropathy. Antibody to sympathetic nerve growth factor was the subject of E. Zaimis's paper; such immunosympathectomy is clearly a valuable tool for studying autonomic functions.

Several fruitful lines of neurochemical research were surveyed. A. N. Davison demonstrated differential incorporation of components into developing myelin and considered that movement of lipoprotein units within the sheath might explain such findings. After this examination of the brain's myelin fraction, V. P. Whittaker surveyed the synaptosome fraction: he showed that acetylcholine is synthesized in synaptic vesicles, whereas cholinesterase activity is sited on the synaptosome's external membrane. Biochemical lesions were next discussed. W. N. Aldridge outlined an elegant method for elucidating the reaction of organophosphorus compounds with certain brain protein fractions. The neurological manifestations of hydroxocobalamin deficiency, Leber's disease and tropical malnutrition were attributed by J. Wilson to different forms of impaired cyanide detoxication.

In the developing tadpole's cord, A. Hughes showed that some 85 per cent of motor cells atrophy; he inferred that such cells fail to make functional peripheral connexions. Turning to the nutritional aspects of brain development, J. Dobbing established that myelin and possibly other elements fail to "catch up" if the grain is undernourished during the early vulnerable period of its development. Concluding this subject, M. Berry and J. T. Eayrs portrayed morphological and behavioural changes after irradiation and hormonal injury to the developing brain.

\section{Phage Coat Protein}

\section{from a Correspondent in Cell Biology}

THE entire genome of the closely related RNA bacteriophages R17, f2, MS2 and fr consists of a single stranded RNA molecule with sufficient information to code for about 1,100 amino-acids. This RNA molecule has the dual function of providing a store for the genetic information and also acting as a messenger. Zinder and his co-workers (1966) and Gussin (1966) have shown that the genomes of $\mathrm{f} 2$ and $\mathrm{R} 17$ have at 